\title{
Transaction
}

\section{Mechanical Properties of Yarns Dyed by Pigments}

\author{
Mari Inoue $^{* 1, \#}$, Nasil Fang ${ }^{* 1}$, Hiroyuki Fujita ${ }^{* 2}$, Yasushi Saeki ${ }^{* 2}$, and Minoru Furutani ${ }^{* 2}$ \\ ${ }^{* 1}$ Graduate School of Human Development and Environment, Kobe University, \\ 3-11 Tsurukabuto, Nada-ku, Kobe, Hyogo 657-8501, Japan, \\ ${ }^{* 2}$ Hyogo Prefecture Institute of Technology, 1790-496 Nomura-cho, Nishiwaki, Hyogo 677-0054, Japan
}

\begin{abstract}
Furutani et al. [1-3] developed a technology for constructing trial fabrics. This technology consists of the dyeing technology realizing low cost and short term production, and minimizing loss of yarn and amount of drainage. Recently, an apparatus for dyeing with pigments were newly developed, in which the dyeing term was shortened by one -ninth, and the amount of drainage was reduced by one-tenth by the new apparatus. In this study, the mechanical properties of the dyed yarn with pigments by the new apparatus were investigated. The characteristics of yarns dyed with pigments were within the $\pm 4 \sigma$ of regular yarn group and there was no difference from the yarns dyed with dye stuffs if the concentration of binder was not beyond 10\%. Therefore, it makes clear that dyed yarn by using the new apparatus can be utilized for trial fabrics.
\end{abstract}

(Received 11 June, 2012; Accepted 20 August, 2012)

\section{Introduction}

Textile companies are subjected to produce an enormous amount of trial fabrics to meet the various requirements of consumer. Without trial fabrics, it is incomplete to check the pattern, design, touch feeling etc. In Japan, it seems that it takes three times of costs and term for manufacturing "trial fabrics" against China. In the meantime, fabric exports of Japan have decreased very much, and foreign countries such as China and Southeast Asian Nations have taken the place of Japan. Thus, Japanese textile producers have been bothered by the high cost and the long manufacturing term for preparing the trial fabrics. Then it follows that the development of processing technology for manufacturing the trial fabrics, which is low costs, short term production, and high quality, is desirable. Besides, the technology should be harmonious with the preservation of the environment.

Furutani et al. [1-3] developed a new system for constructing the trial fabric system. The system consists of the following three technologies, that is, 1 : dyeing technology enabling low-cost and short-term production, and minimizing loss of yarn and amount of drainage, 2 : recycling technology of the remainder of dyed yarn, and 3 : new filling insertion technology.

In usual dyeing processes, the cheese or beam dye by

\# corresponding author batch dyeing are done by using chemical dyestuff. However, it takes long times and it needs a lot of water and chemicals. In this new technology of producing trial fabrics, a new apparatus for dyeing one single yarn by pigments were developed, and dyeing term is shortened by one-ninth, amount of drainage is reduced by one-tenth. In this study, we investigate the mechanical properties of the dyed yarn with pigments by the new dyeing technology.

\section{Experimental}

\subsection{Samples}

Each sample single yarn was dyed by the 2dip-2nip method (dipped and nipped process in twice) by using the dyed machine (KS-7 Unisizer, Kaji Seisakusho Co. Ltd.). The dyeing condition was as follows: temperature was room temperature $\left(25^{\circ} \mathrm{C}\right)$, dyeing speed was $80 \mathrm{~m} / \mathrm{min}$. The dyed samples were dried at $80^{\circ} \mathrm{C}$ for three minutes.

Nineteen cotton yarn samples dyed by pigments were used in this study. The sample details were shown in Table 1. As a reference, Y0 sample before dyeing was prepared. All the all yarn samples were 40's cotton counts. In the case of Y1-Y10 samples, the concentration of pigments (Red, Mitsubishi Pencil Co., Ltd.) in water was fixed at $5 \%$, and the concentration of binder for each sample was changed.

For the samples of Y11-Y19, the concentration of binder (PO-0, Mitsubishi Pencil Co., Ltd.) was fixed at 
$5 \%$ and the concentration of pigment (Yellow and Red, Mitsubishi Pencil Co., Ltd.) was changed. The samples, Y15 and Y19, were finished using a silicone softener (High Softener KZ-3M, Meisei Chemical Works, Ltd.).

Two samples dyed by dyestuffs, of which colors were red and navy, and bleached sample (white) were added.

Table 1 Samples details, the concentration of pigment, binder and softener.

\begin{tabular}{ccccl}
\hline Sample & $\begin{array}{c}\text { Pigment } \\
\%\end{array}$ & $\begin{array}{c}\text { Binder } \\
\%\end{array}$ & $\begin{array}{c}\text { Softener } \\
\%\end{array}$ & remarks \\
\hline Y0 & 0 & 0 & 0 & before dyeing \\
\hline Y1 & 5 & 1 & 0 & Color: Red \\
Y2 & 5 & 2 & 0 & \\
Y3 & 5 & 3 & 0 & \\
Y4 & 5 & 4 & 0 & \\
Y5 & 5 & 5 & 0 & \\
Y6 & 5 & 7 & 0 & \\
Y7 & 5 & 10 & 0 & \\
Y8 & 5 & 15 & 0 & \\
Y9 & 5 & 20 & 0 & \\
Y10 & 5 & 30 & 0 & \\
\hline Y11 & 0.5 & 5 & 0 & Color: Yellow \\
Y12 & 1 & 5 & 0 & \\
Y13 & 3 & 5 & 0 & \\
Y14 & 5 & 5 & 0 & \\
Y15 & 5 & 5 & 1 & \\
\hline Y16 & 0.5 & 5 & 0 & Color: Red \\
Y17 & 1 & 5 & 0 & \\
Y18 & 3 & 5 & 0 & \\
Y19 & 5 & 5 & 1 & \\
\hline & & & & \\
\hline
\end{tabular}

\subsection{Characterization of yarns}

Tensile, torsion, bending, compression and surface properties of yarns were measured for the characterization of yarns [4-11]. The measurement parameters and condition are listed in Table 2 and 3.

Tensile property was measured by tensile tester KES-G5-TH (Kato Tech Co., Ltd.). Tensile tester and an
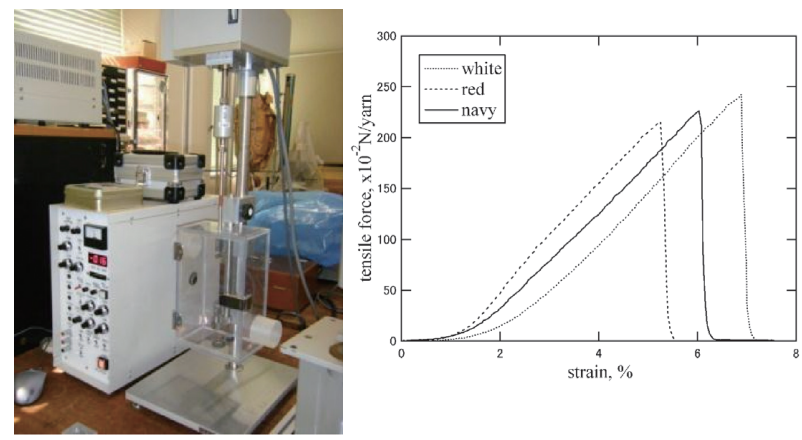

Fig. 1 Tensile tester, KES-G5-TH and tensile properties of cotton yarns dyed by dyestuff. example of the force-strain properties of cotton yarns at constant rate of extension are shown in Fig. 1.

A piece of yarn approximately $100 \mathrm{~mm}$ in length was reinforced at both ends by gluing pieces of paper to the ends, so that the yarn could be clamped by the chucks of a tensile tester. A constant strain rate of $0.4 \%$ per second was applied to test the tensile strength of yarn. After the tensile force attains at maximum force of $0.15 \mathrm{~N} / \mathrm{tex}$, the recovery process was also measured and linearity $(L T)$, tensile energy per unit area $(W T)$ and resilience $(R T)$ were obtained [4-6]. The breaking force $(B F)$ and strain

Table 2 Characterization of yarn samples.

\begin{tabular}{|c|c|c|}
\hline Property & Parameter & \\
\hline \multirow[t]{5}{*}{ Tensile } & $L T(-)$ & Linearity \\
\hline & $W T(N m / m . y a r n)$ & Energy \\
\hline & $R T(\%)$ & Resilience \\
\hline & $B E(-)$ & Breaking strain \\
\hline & $B F(N)$ & Breaking Force \\
\hline \multirow[t]{4}{*}{ Torsion } & $G P(\mathrm{mN} /$ rad.yarn $)$ & Rigidity (+) \\
\hline & GN(mN/rad.yarn) & Rigidity (-) \\
\hline & $G M(m N /$ rad.yarn $)$ & Means of rigidity \\
\hline & $2 H G(\mathrm{mN} /$ yarn $)$ & Hysteresis \\
\hline \multirow[t]{2}{*}{ Bending } & $B(N \cdot \mathrm{m} 2 /$ yarn $)$ & Rigidity \\
\hline & $2 H B(\mathrm{Nm} /$ yarn $)$ & Hysteresis \\
\hline \multirow[t]{3}{*}{ Compression } & $L C(-)$ & Linearity \\
\hline & $W C\left(N m / m^{2}\right)$ & Energy \\
\hline & $R C(\%)$ & Resilience \\
\hline \multirow{3}{*}{$\begin{array}{l}\text { Compression } \\
\text { (Wire } \\
\text { method) }\end{array}$} & $L C w(-)$ & Linearity \\
\hline & $W C w(\mathrm{Nm} / \mathrm{m})$ & Energy \\
\hline & $R C w(\%)$ & Resilience \\
\hline \multirow[t]{3}{*}{ Surface } & $M I U(-)$ & $\begin{array}{l}\text { Mean of } \\
\text { coefficient of } \\
\text { friction }\end{array}$ \\
\hline & $M M D(-)$ & $\begin{array}{l}\text { Mean deviation of } \\
\text { coefficient of } \\
\text { friction }\end{array}$ \\
\hline & $S M D(\mu \mathrm{m})$ & $\begin{array}{l}\text { Mean deviation of } \\
\text { surface roughness }\end{array}$ \\
\hline
\end{tabular}

Table 3 Measurement condition.

\begin{tabular}{ll}
\hline Property & Measurement condition \\
\hline Tensile & $\begin{array}{c}\text { Fmax }=0.15 \mathrm{~N} / \text { tex } \\
\text { and under breaking }\end{array}$ \\
& $\begin{array}{l}\text { length }=100 \mathrm{~mm} \\
\text { strain speed }=0.4 \% / \mathrm{sec}\end{array}$ \\
\hline Torsion & $\begin{array}{l}\text { torsion angle }=+2 \pi \\
\text { speed }=\pi / 2 / \mathrm{sec} \\
\text { length }=10 \mathrm{~mm}\end{array}$ \\
& $\begin{array}{l}\text { length }=10 \mathrm{~mm} \\
\text { curvature }=+2.5 \mathrm{~cm}^{-1}\end{array}$ \\
\hline Bending & Fmax $=0.05 \mathrm{mN} / \mathrm{m}^{2}$ \\
\hline Compression & \\
\hline Compression & Fmax $=0.0725 \mathrm{~N} / \mathrm{mm} / \mathrm{tex}$ \\
(Wire method) & \\
\hline Surface & $\mathrm{P}=0.5 \mathrm{~N}$ \\
\hline
\end{tabular}


$(B E)$ were obtained from the strain-force curve at the point of fracture.

The torsion property of yarn was measured by torsion tester [6,7]. A constant rate of torsion was applied at a rate of $0.2 \pi$ radian per second to a yarn specimen, using the yarn torsion tester. The sample length was approximately $10 \mathrm{~mm}$. A constant tension was applied by $0.02 \mathrm{~N}$ weights during measurement. The maximum torsion angle is $\pm 0.2 \pi / \mathrm{mm}$. Four characteristic parameters: the torsion rigidities for increasing and decreasing twist $(G P$ and $G N)$; the average torsional rigidity $(G M)$; and the hysteresis of torque at a torsion angle of zero $(2 H G)$, were obtained from the curve. A typical torque-torsion angle relation for cotton yarns dyed by dyestuff is shown in Fig. 2 with the torsion tester.
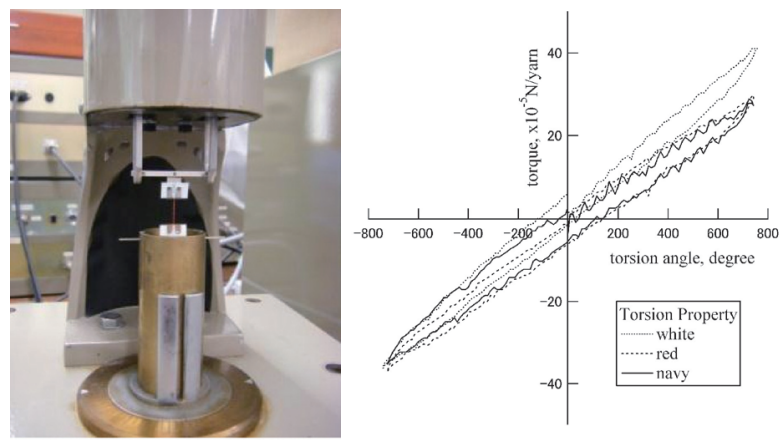

Fig. 2 Torsion tester and the torsion properties of cotton yarns dyed by dyestuff.

The bending property was measured by bending tester shown in Fig. 3. The 20 yarns were fixed at intervals of $1 \mathrm{~mm}$ in $25 \mathrm{~mm}$ length and $10 \mathrm{~mm}$ in width, and this $10 \mathrm{~mm}$ width was bent as shown in Fig. 3. Pure bending between the curvatures $\mathrm{K}=-2.5$ and $2.5\left(\mathrm{~cm}^{-1}\right)$ with constant rate of curvature change. The rate was 0.50 $\mathrm{cm}^{-1} / \mathrm{sec}$. Characteristic values were bending rigidity $(B)$ and moment of hysteresis $(2 H B)$ per yarn $[4,6,8]$,.
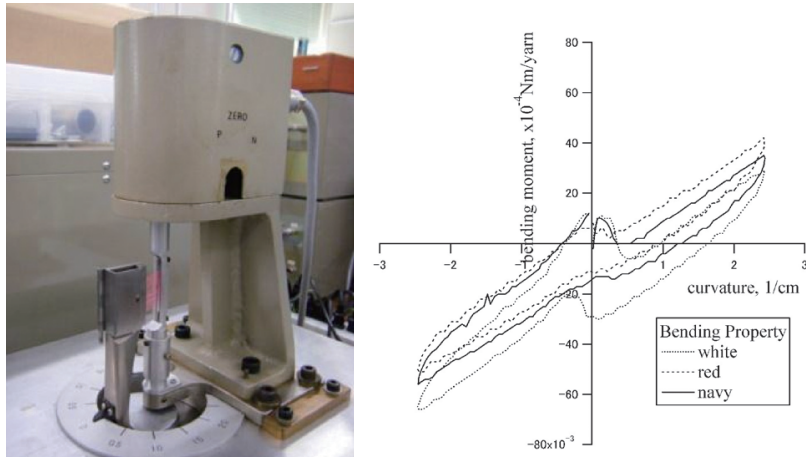

Fig. 3 Bending tester and the bending properties of cotton yarns dyed by dyestuff.

The compression property of yarn was measured by two methods. One method was that the specimen fixed at intervals of $1 \mathrm{~mm}$ was compressed by two circular-plates of steel having $2 \mathrm{~cm}^{2}$ area by using compression tester, KES-FB3 [4]. The velocity of the compression was $20 \mu \mathrm{m} / \mathrm{sec}$ and when the pressure attains at $0.05 \mathrm{mN} / \mathrm{m}^{2}$, the recovery process was measured by the same velocity. As the characteristics values, linearity $(L C)$, compression energy $(W C)$ and resilience $(R C)$ were obtained.

The other method was the compression of the side of one yarn. The changes in yarn thickness caused by tension were measured using the wire method [4-6,8-10] as shown in Fig. 4. A piece of yarn was hung on the tension $\operatorname{arm} F_{y}$. The angle between the yarn and the horizontal level was $30^{\circ}$, which was approximately equal to the average yarn intersecting angle in various weaves. The yarn thickness at the crossover point was measured by a linear differential transducer, having a needle sensor that was contacting at the top of the yarn surfaces with a small compressional force, and the thickness was recorded as a function of yarn tension. The yarn tension was detected by a force transducer and recorded. Three characteristic parameters, linearity $(L C w)$, compression energy $(W C w)$ and resilience $(R C w)$ were obtained.

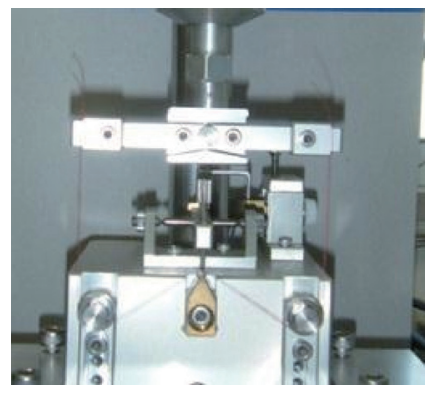

Fig. 4 Compression tester (wire method)

Surface friction and roughness of a single yarn were measured by surface analyzer as shown in Fig. 5 .

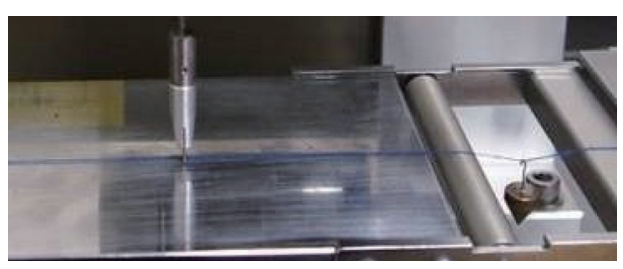

Fig. 5 Surface tester

The contactor for measurement of surface friction and roughness was made by a piano wire of which diameter is $0.5 \mathrm{~mm}$. The wire was bent as $U$ shape, and used under the contact force of $0.1 \mathrm{~N}$. The specimen was moved between $20 \mathrm{~mm}$ interval by a constant velocity of $1 \mathrm{~mm} / \mathrm{sec}$ on a smooth steel plate placed horizontally and the contactor is kept its position. Three characteristic values [4], mean value of the coefficient of friction $(M I U)$, mean deviation of coefficient of friction $(M M D)$ 
and mean deviation of surface roughness (SMD).

The mechanical and surface properties of ten to fifteen yarns and bending property of sixteen yarns were measured and the mean value and standard deviation of each property were calculated.

\section{Results and discussion}

It is useful to express the characteristic values on a chart to inspect the property of the yarn and its characterization. The scales of the horizontal axis are normalized by the mean value and the standard deviation of each of corresponding characteristic values. It is noted that some of the characteristic values are normalized by taking their logarithm values because these characteristic values are shown by their logarithm to obtain a symmetric shape of the distribution curve [4]. The mean value $M_{i}$ ( $i=1-20)$, and standard deviation, $\sigma_{i}(i=1-20)$ of the mechanical and surface properties $X_{i}(i=1-20)$ of yarns before dyeing and dyed yarn by dyestuffs, of which the yarn counts are 40's are shown in Table 4. Fig. 6 shows the characterization of one yarn before dyeing, bleached yarn (called white yarn) and yarns dyed by red and navy dyestuffs on the chart. The common scale is the scale normalized by the standard deviations, $\left(X_{i}-M_{i}\right) / \sigma_{i}(i=1$ 20). And various mechanical and surface properties of a yarn could be explained and defined the property of the yarn by the chart.

Table 4 The mean value and the standard deviation of 40's cotton yarn.

\begin{tabular}{cccc}
\hline$i$ & $X_{i}$ & Mean, $M_{i}$ & $\mathrm{SD}, \sigma_{i}$ \\
\hline 1 & $L T$ & 0.5862 & 0.0420 \\
2 & $\log W T$ & -1.5378 & 0.1213 \\
3 & $R T$ & 57.9149 & 4.2554 \\
4 & $\log E B$ & -1.2694 & 0.1095 \\
5 & $\log F B$ & 2.7336 & 0.0306 \\
\hline 6 & $\log B$ & -1.8151 & 0.1836 \\
7 & $\log 2 H B$ & -2.0116 & 0.2163 \\
\hline 8 & $\log G P$ & 0.6609 & 0.1657 \\
9 & $\log G N$ & 0.5454 & 0.1118 \\
10 & $\log G M$ & 0.6280 & 0.0878 \\
11 & $\log 2 H G$ & 0.1871 & 0.2329 \\
\hline 12 & $L C$ & 0.2435 & 0.0491 \\
13 & $\log W C$ & -0.6261 & 0.2752 \\
14 & $R C$ & 45.7309 & 6.0711 \\
\hline 15 & $L C w$ & 0.1935 & 0.0312 \\
16 & $\log W C w$ & -0.9949 & 0.0369 \\
17 & $R C w$ & 20.7546 & 5.4431 \\
\hline 18 & $M I U$ & -0.8366 & 0.0625 \\
19 & $\log M M D$ & -2.1275 & 0.0769 \\
20 & $\log S M D$ & 0.3627 & 0.1206 \\
\hline & & &
\end{tabular}

In the case of mechanical and surface properties of fabrics for the same usage, the distribution of each property is almost located within $-4 \sigma$ to $4 \sigma$ [4]. Because the yarn property is of uneven quality in comparison with the fabric property, it is considered that the properties of yarn which are located within $-4 \sigma$ to $4 \sigma$ show the similar performance.

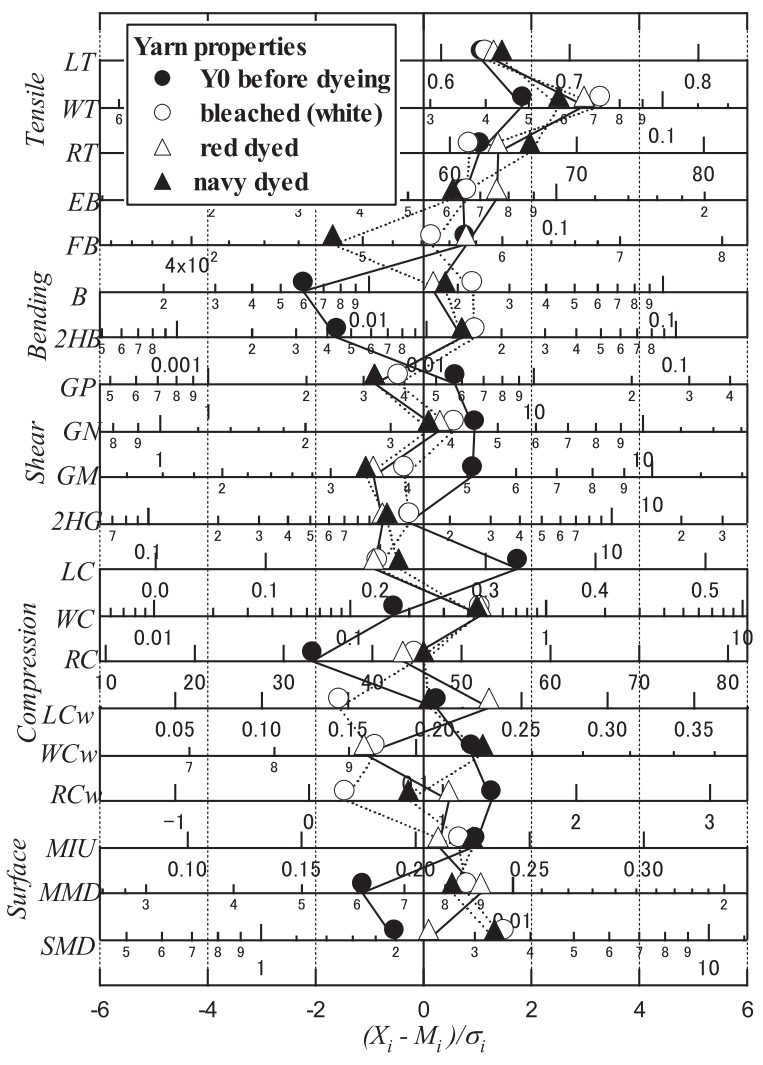

Fig. 6 The characterization of yarns before dyeing and the yarns dyed by dyestuff on the chart.

The characterization of yarns dyed by dyestuff used in this experiment is located within $-2 \sigma$ to $+2 \sigma$. It seems that the performance of yarns depends on dyeing or not, and the color of dyestuff. Although the chart is only used for expressing the fabric characterization, it makes clear that the method also applied the characterization of yarns.

Fig. 7 and Fig. 8 show the characterization of sample yarns dyed by pigments, for which the concentration of binder was changed, on the chart. Y1, Y3 and Y5 samples showed the similar properties as shown in Fig. 7. The bending and torsional properties of Y5 were a little larger. However, their properties of $\mathrm{Y} 7$ having $10 \%$ binder concentration were one or two sigma larger than Y1, Y3 and Y5. Their properties of Y8, Y9 and Y10 were bigger than Y7. The concentration of binder was within $10 \%$ in Fig. 7, and beyond $10 \%$ in Fig. 8. The characteristics of yarns dyed by pigments with $10 \%$ binder concentration were located within $-4 \sigma$ to $+4 \sigma$. However, the bending and torsion properties of yarns for which the 


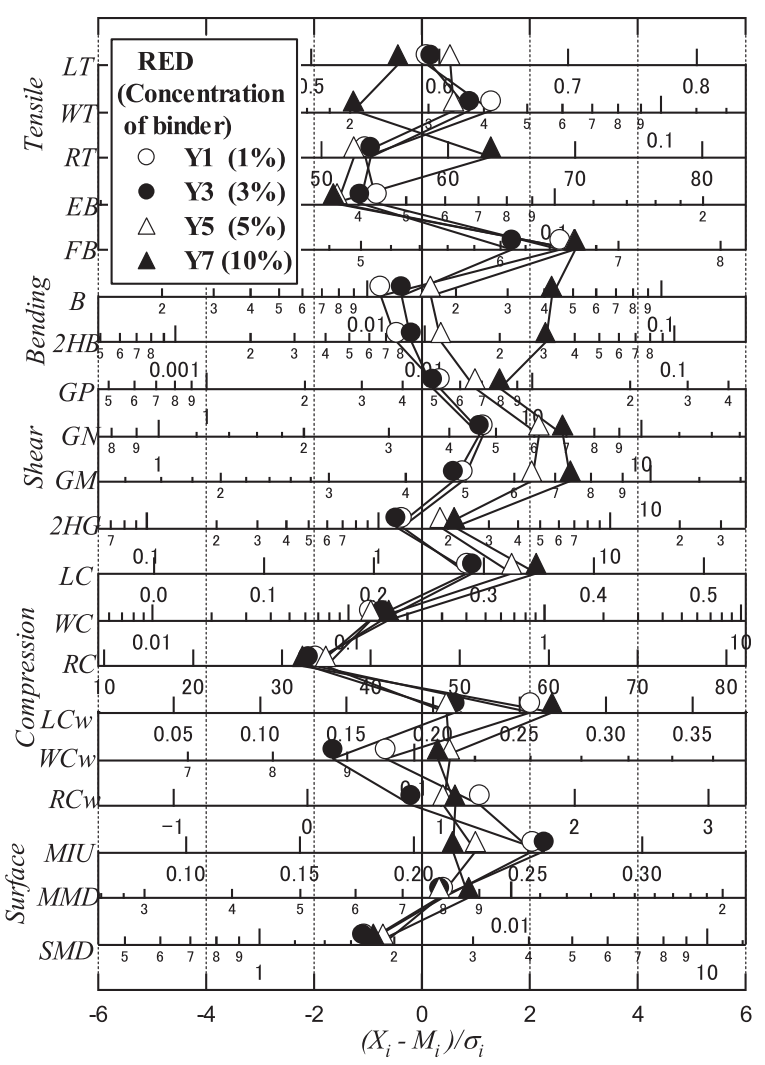

Fig. 7 The characterization of yarns dyed by pigments which the concentration of binder is within $10 \%$.

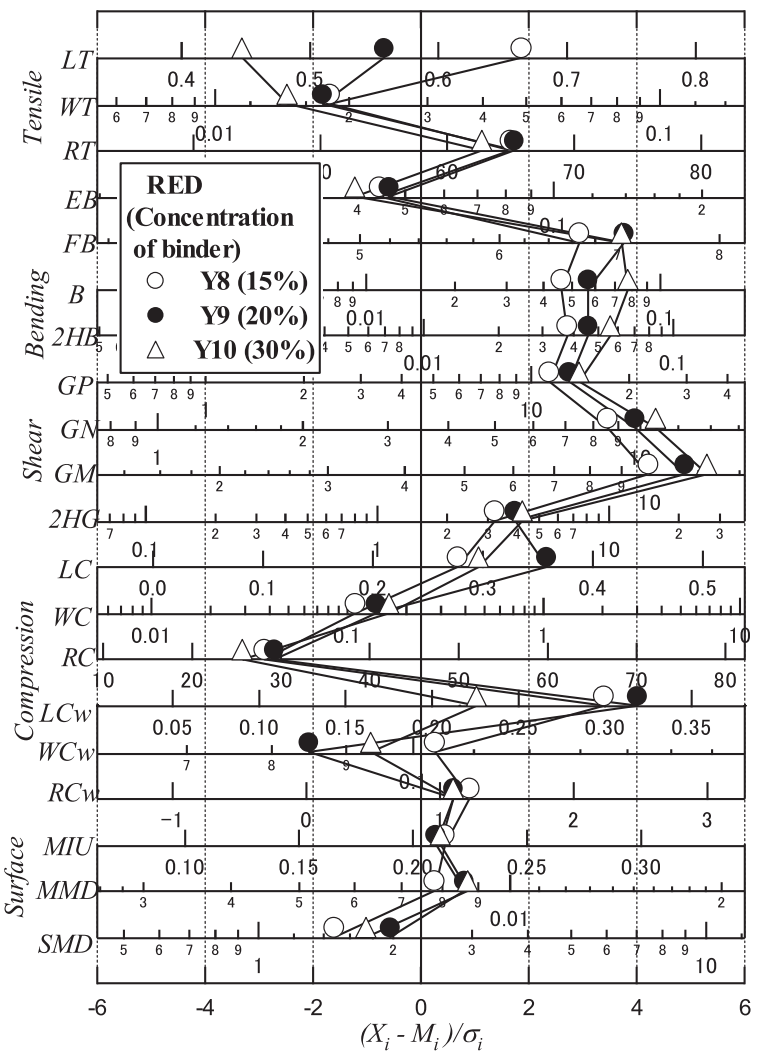

Fig. 8 The characterization of yarns dyed by pigments which the concentration of binder is beyond $10 \%$.

concentration of binder was beyond $10 \%$ increased and were beyond $+5 \sigma$.
In the case of the fabrics, the characteristics values of the same usage were located within about $-4 \sigma$ to $+4 \sigma$ as stated above. Compared with the fabrics, the ranges of the yarn properties vary widely. Therefore, it seems that these yarns dyed by pigments can easily stand comparison with the yarns dyed by dyestuff. It showed that the stiffness of yarns for which the concentration of binder was beyond $10 \%$ were larger than the yarns dyed by dyestuffs.

Fig. 9 and Fig.10 show the change of the normalized value of bending and torsion parameters by the binder concentration. These parameters increased, indicating that the bending and torsion rigidity of the yarns became stiff with increasing of the binder concentration.

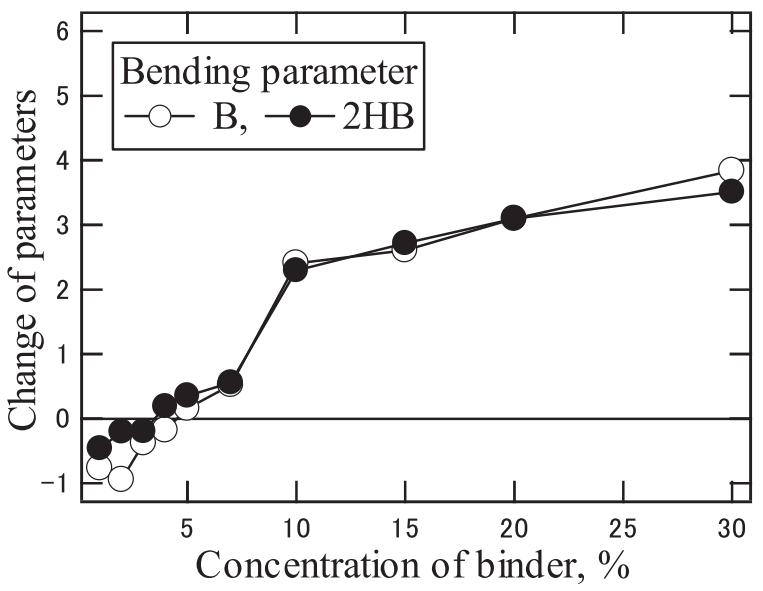

Fig. 9 The change of the bending parameters by the concentration of binder

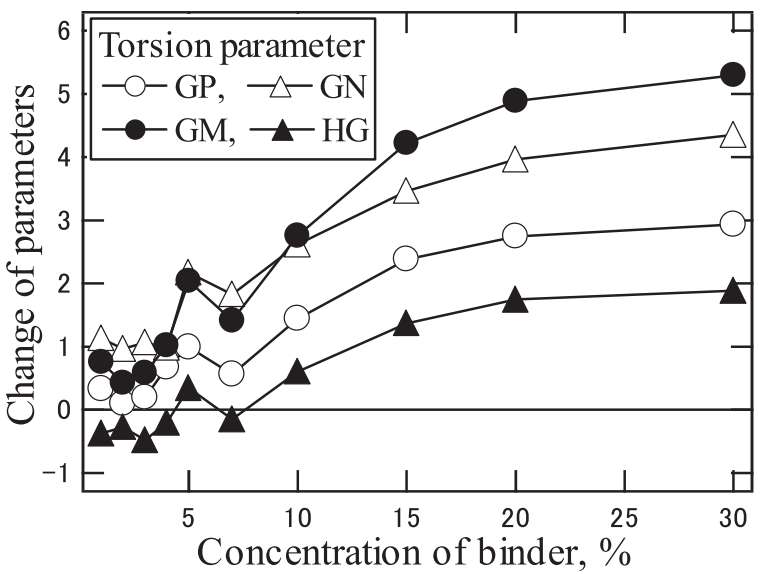

Fig. 10 The change of the torsion parameters by the concentration of binder.

Fig. 11 and Fig. 12 show the characterization of sample yarns dyed by yellow and red pigments, respectively, for which the concentration of pigment was changed on the chart. It had the tendency that $R T$ and $R C$ of the tensile and compression properties were decreased and $2 H B$ and $2 H G$ of the bending and torsion properties were increased. 


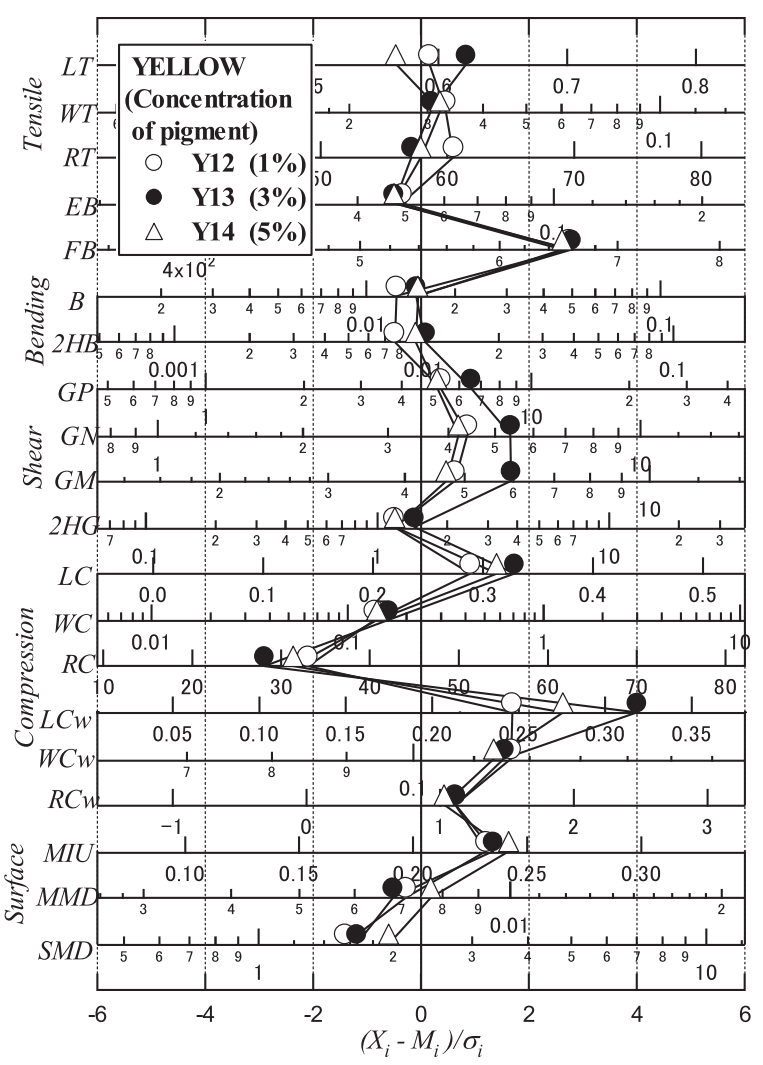

Fig. 11 The characterization of yarns dyed by pigments which the concentration of yellow pigment were changed.

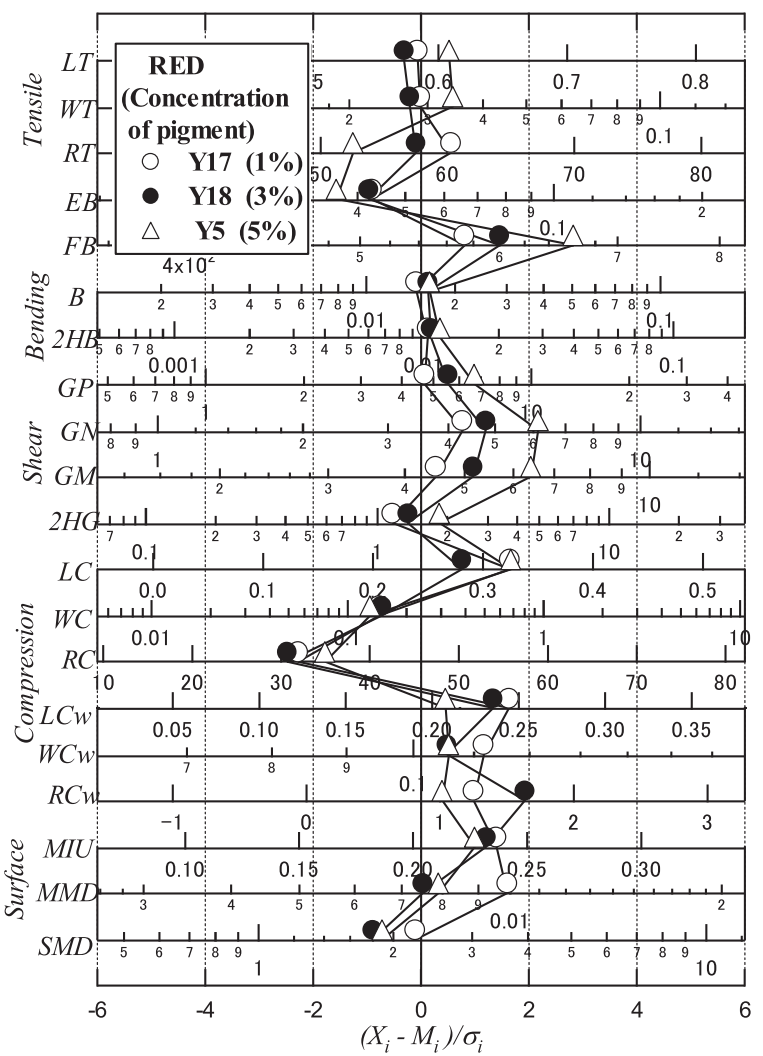

Fig. 12 The characterization of yarns dyed by pigments which the concentration of red pigment were changed.
The change of the normalized value of bending properties by the change of the concentration of pigment was shown in Fig. 13.
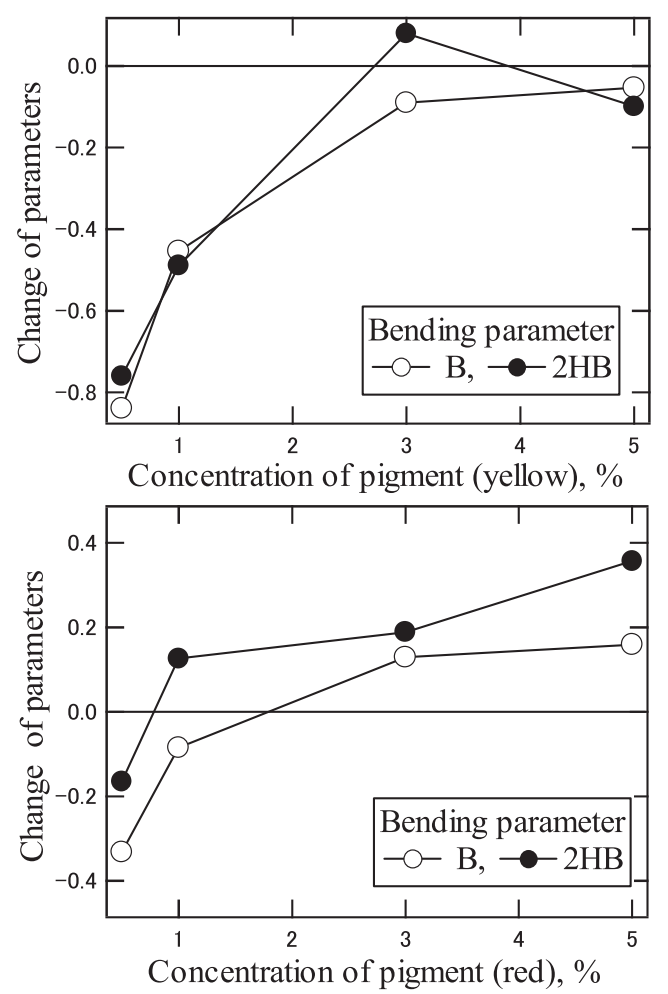

Fig. 13 The change of the bending parameters of yarns dyed by pigments which the concentration of pigment were changed.

The change of the normalized value of torsion and surface properties by the change of the concentration of pigment is shown in Fig. 14 and Fig. 15, respectively. In both the red and yellow pigments, the increasing of the pigment concentration caused the increasing of the bending and torsion properties, and $M M D$ and SMD of surface property.

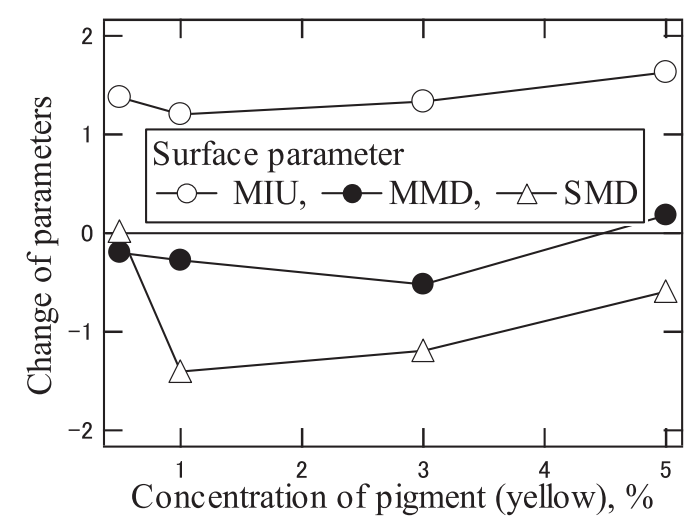

Fig. 14 The change of the surface parameters of yarns dyed by pigments where the pigment concentration were changed. 


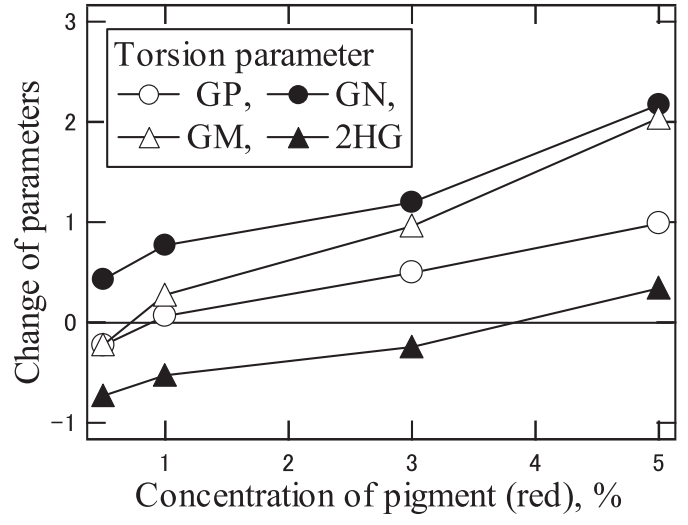

Fig. 15 The change of the torsion parameters of yarns dyed by pigments where the pigment concentration were changed.

Fig. 16 and Fig. 17 show the characterization of sample yarns dyed by pigments with silicon on the chart. These figures show the effects of the addition of silicon softener. By the addition of silicon, breaking force in tensile deformation, bending and torsion parameters and $M M D$ of surface properties became smaller. It is known that the addition of softener to fabric makes the decrease of $2 H B$ and $2 H G, M M D$ and $S M D$ [12]. The results showed the similarity with the yarn properties.

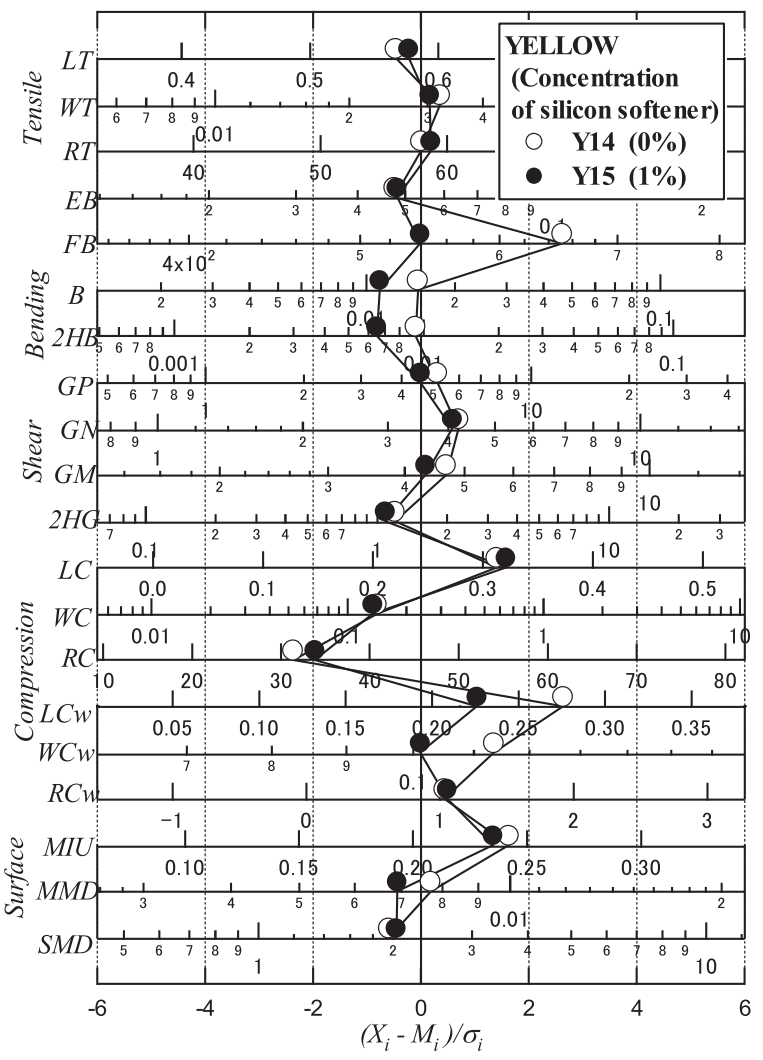

Fig. 16 The change of the torsion parameters of yarns dyed by pigments where the pigment concentration were changed.

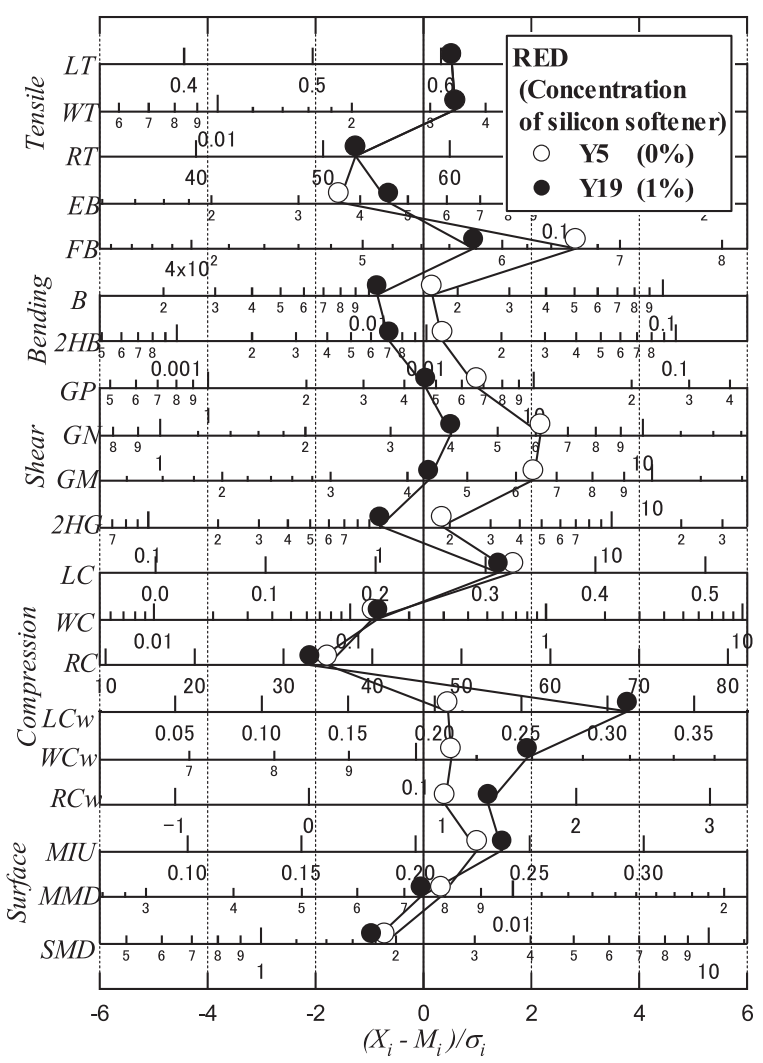

Fig. 17 The characterization of yarns dyed by yellow pigment with silicon softener on the chart.

\section{Conclusions}

The mechanical properties of the dyed yarn by pigments were investigated in comparison to the properties of dyed yarn by dyestuffs. As the results, the following contents are clarified.

The increasing of the concentration of binder and pigment causeed the increasing bending and torsion parameters. The addition of softener was caused the decreasing breaking force, bending and torsion parameter, $M M D$. The characterizations of yarns dyed by pigments were within the $\pm 4 \sigma$ of regular yarn group and there was no difference from the yarns dyed by dye stuffs, if the concentration of binder exceeded $10 \%$. Thus, it makes clear that dyed yarn by using the new method can be utilized for trial fabric.

In this study, mechanical and surface properties measurements of yarns were operated as like that of textile fabrics. Tensile, torsion, bending, compression and surface properties of yarns were measured for the characterization of yarns to define "properties of yarns"

\section{References}

1. M.Furutani et al., Reports of support industry (20072009). 
2. S. Katayama, H. Fujita, M. Furutani et al., Proceedings of the 62nd Annual Meeting of The Text. Mach. Soc. of Japan, 202-205 (2009).

3. M. Furutani, Proceedings of the $17^{\text {th }}$ Autumn Seminar of The Text. Mach. Soc. of Japan, 53-60 (2010).

4. S. Kawabata, "The Standardization and Analysis of Hand Evaluation (2ed.)", The HESC and The Text. Mach. Soc. of Japan (1980).

5. M. Inoue, S. Yamamoto, Y.Yamada and M. Niwa, Text. Res. J., 76, 534-539 (2006).

6. M. Niwa and S. Kawabata; Theoretical analysis of the tensile property of yarn, Text. Mach. Soc. J., 28, T153-156 (1975).

7. S. Kawabata, M. Niwa and T. Mamiya ; Torsional testing apparatus and its application to experimental investigation on the relation between torsional properties of yarns and fibers, Text. Mach. Soc. J., 29, T119-131 (1976).

8. M. Niwa and S. Kawabata; Bending rigidity of the yarn with inter-fiber friction induced by lateral compressive force, Text. Mach. Soc. J., 27, T192200 (1974).
9. M. Niwa and S. Kawabata; On the residualinteracting force at the crossing point of warp and weft of plain-weave fabrics, Text. Mach. Soc. J., 26, T208-216 (1973).

10. M. Niwa and S. Kawabata; Theoretical analysis of the large deflection for the elastic yarn with interfiber friction, Text. Mach. Soc. J., 28, T146-152 (1975) .

11. M. Niwa, R. Sugishita and S. Kawabata; Corona discharge treatment of polyester spun-yarn for fixing lubricant on the yarn surface for the purpose of decreasing its frictional coefficient, Text. Mach. Soc. J., 27, T208-214 (1974).

12. M. Inoue, Y. Sano, M. Uyama, and M. Niwa; Effects of softener treatment on hand, thermal and moisture transport and wear comfort of knitted fabrics, Sen'i gakkaishi, 53, 226-238 (1997). 